\title{
Mutations in $\gamma$-secretase subunit-encoding PSENEN underlie Dowling-Degos disease associated with acne inversa
}

\author{
Damian J. Ralser, ${ }^{1}$ F. Buket Ü. Basmanav, ${ }^{1}$ Aylar Tafazzoli, ${ }^{1}$ Jade Wititsuwannakul, ${ }^{2}$ Sarah Delker, ${ }^{3}$ Sumita Danda, ${ }^{4}$ Holger Thiele, ${ }^{5}$ \\ Sabrina Wolf, ${ }^{1}$ Michélle Busch, ${ }^{6}$ Susanne A. Pulimood, ${ }^{7}$ Janine Altmüller, ${ }^{5}$ Peter Nürnberg, ${ }^{5}$ Didier Lacombe, ${ }^{8}$ Uwe Hillen, ${ }^{3}$ \\ Jörg Wenzel, ${ }^{9}$ Jorge Frank, ${ }^{10}$ Benjamin Odermatt, ${ }^{6}$ and Regina C. Betz ${ }^{1}$ \\ IInstitute of Human Genetics, University of Bonn, Bonn, Cermany. ${ }^{2}$ Department of Medicine, Chulalongkorn University, Bangkok, Thailand. ${ }^{3}$ Department of Dermatology, University of Essen, Essen, Germany. \\ ${ }^{4}$ Department of Clinical Genetics, Christian Medical College and Hospital, Tamil Nadu, India. ${ }^{5}$ Cologne Center for Genomics, University of Cologne, Cologne, Germany. ${ }^{6}$ Institute of Anatomy, University of Bonn, \\ Bonn, Germany. ${ }^{7}$ Department of Dermatology, Christian Medical College and Hospital, Tamil Nadu, India. ${ }^{8}$ Department of Cenetics, Centre Hospitalier Universitaire (CHU) de Bordeaux, Bordeaux, France. \\ ${ }^{9}$ Department of Dermatology, University of Bonn, Bonn, Germany. ${ }^{10}$ Department of Dermatology, Venereology and Allergology, University Medical Center Cöttingen, Göttingen, Germany.
}

Dowling-Degos disease (DDD) is an autosomal-dominant disorder of skin pigmentation associated with mutations in keratin 5 (KRT5), protein 0-fucosyltransferase 1 (POFUT1), or protein 0-glucosyltransferase 1 (POCLUT1). Here, we have identified 6 heterozygous truncating mutations in PSENEN, encoding presenilin enhancer protein 2, in 6 unrelated patients and families with DDD in whom mutations in KRT5, POFUT1, and POCLUT1 have been excluded. Further examination revealed that the histopathologic feature of follicular hyperkeratosis distinguished these 6 patients from previously studied individuals with DDD. Knockdown of psenen in zebrafish larvae resulted in a phenotype with scattered pigmentation that mimicked human DDD. In the developing zebrafish larvae, in vivo monitoring of pigment cells suggested that disturbances in melanocyte migration and differentiation underlie the DDD pathogenesis associated with PSENEN. Six of the PSENEN mutation carriers presented with comorbid acne inversa (AI), an inflammatory hair follicle disorder, and had a history of nicotine abuse and/or obesity, which are known trigger factors for AI. Previously, PSENEN mutations were identified in familial Al, and comanifestation of DDD and AI has been reported for decades. The present work suggests that PSENEN mutations can indeed cause a comanifestation of DDD and Al that is likely triggered by predisposing factors for AI. Thus, the present report describes a DDD subphenotype in PSENEN mutation carriers that is associated with increased susceptibility to Al.

\section{Introduction}

Skin pigmentation is a complex process in which the location, degree, and type of pigmentation provides a visual manifestation of genetic heterogeneity in pathways regulating the function of melanocytes. Melanocytes are located predominantly in the epidermis or hair follicles of the skin. Here, they produce melanin in melanosomes, which are transferred to the cytoplasm of keratinocytes. Skin pigmentation is orchestrated by diverse genes and proteins with an involvement in melanin synthesis, as well as melanosome structure, trafficking, and distribution (1).

Hereditary pigmentation disorders are clinically and genetically heterogeneous. Clinically, hyperpigmentation or hypopigmentation can be observed, occasionally with a strong aesthetical impairment.

Dowling-Degos disease (DDD) (Mendelian Inheritance in Man [MIM] nos. 179850, 615327, and 615696) was first described by Dowling (2) and Degos (3). Patients usually present with pro-

Authorship note: B. Odermatt and R.C. Betz contributed equally to this work Conflict of interest: $P$. Nürnberg is a founder, CEO, and shareholder of ATLAS Biolabs $\mathrm{GmbH}$. ATLAS Biolabs $\mathrm{GmbH}$ is a service provider for genomic analyses.

Submitted: September 13, 2016; Accepted: January 17, 2017.

Reference information: / Clin Invest. 2017;127(4):1485-1490.

https://doi.org/10.1172/JCI90667. gressive reticulate hyperpigmentation and small, dark-brown hyperkeratotic papules on the trunk, face, flexures, large skin folds, and extremities. Histologically, DDD is characterized by a filiform epithelial downgrowth of epidermal rete ridges, with an accumulation of melanin at the tips (4).

DDD is autosomal-dominantly inherited, and to date causal mutations in 3 genes have been identified. In 2006, we detected mutations in KRT5, which encodes the intermediate filament protein keratin 5 (4). In 2013, mutations in POFUT1, which encodes protein O-fucosyltransferase 1, were identified (5). In 2014, our group detected mutations in POGLUT1, which encodes protein O-glucosyltransferase 1 (6). POFUT1 and POGLUT1 are involved in the posttranslational modification of Notch proteins.

Here, we identified mutations in PSENEN, which encodes presenilin enhancer protein 2 (PEN-2), a subunit of the $\gamma$-secretase complex, in patients with DDD. Interestingly, approximately half of these individuals also presented with the chronic inflammatory skin disease acne inversa (AI), demonstrating that mutations in PSENEN can cause DDD and, in co-occurrence of trigger factors, also AI. Using mammalian cell culture studies and knockdown experiments of the PSENEN homolog psenen in zebrafish larvae, we subsequently assessed the functional significance of PSENEN mutations in the disease pathogenesis. 

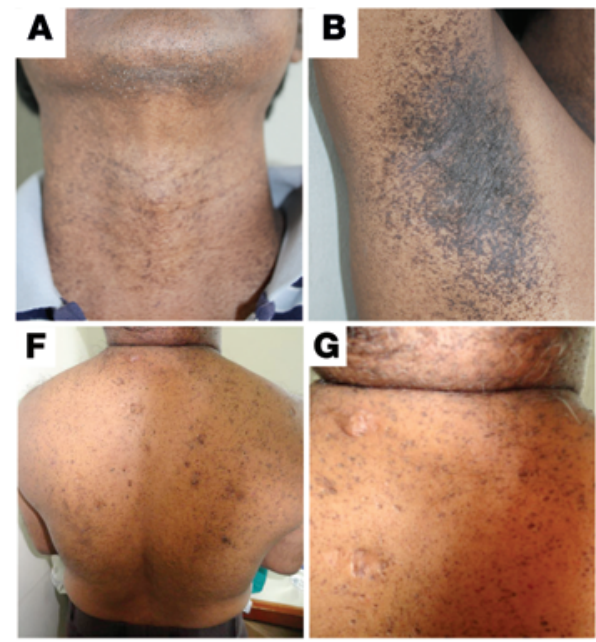

$\mathbf{K}$

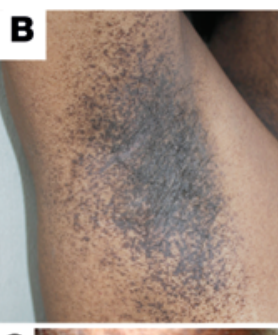

c.35T >A

p.Leu12*
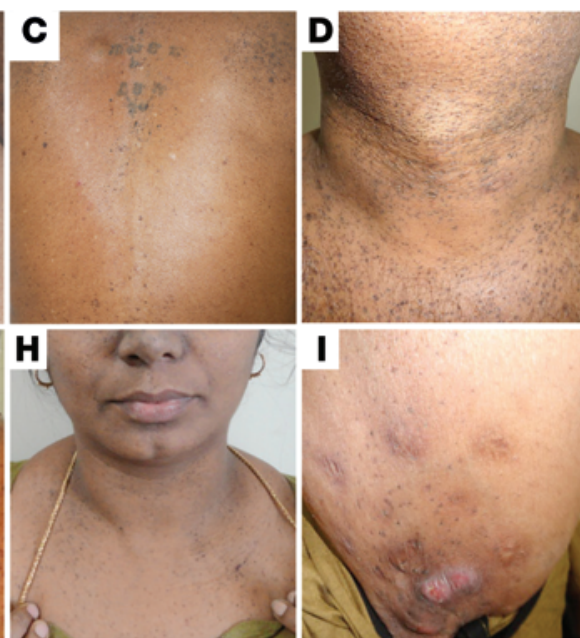

c.84_85ins T

c. $115 \mathrm{C}>\mathrm{T}$

p.Leu29Serfs* 9

p.Arg39*
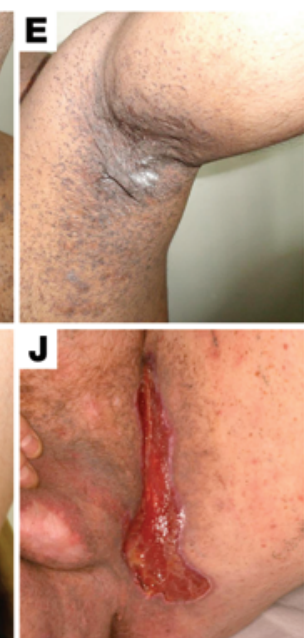

c.216delC

p.Ser73Profs ${ }^{\star} 72$
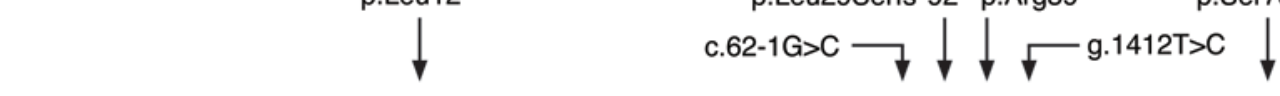

Figure 1. Clinical presentation and location of mutations in PSENEN. (A-C) Familial case in a Thai male with reticulate hyperpigmentation in the (A) frontal aspect of the neck, (B) axilla, and (C) back. (D-I) Affected members of an Indian family. (D) Indian man with reticulate hyperpigmentation and hyperkeratotic brown papules on the neck, $(\mathbf{E})$ axilla, and (F and $\mathbf{G})$ trunk. (E) Al is present in the axillary region. This patient's sister shows (H) hyperpigmentation of the intermammary cleft and (I) Al in the axilla. (J) German man with reticulate hyperpigmentation in the perianal region, which represents a postoperative lesion following surgical treatment for AI. (K) Depiction of the exon structure of PSENEN. Identified mutations are indicated by arrowheads. Mutations are defined at both the nucleotide and protein levels.

\section{Results and Discussion}

Using whole-exome and Sanger sequencing, we detected 6 distinct heterozygous PSENEN mutations in 6 unrelated patients or families with the clinical and histopathological features of DDD, in whom mutations in known DDD genes had been excluded (Figure 1, A-J, Table 1, and Supplemental Figure 2; supplemental material available online with this article; https://doi.org/10.1172/ JCI90667DS1). On closer examination of the histological sections, we identified what to our knowledge is a novel feature that distinguished these individuals from previous DDD cases by the presence of follicular hyperkeratosis (Figure 2, A and B).

PSENEN encodes presenilin enhancer protein 2 (PEN-2), a member of the $\gamma$-secretase enzyme complex, which is responsible for the intracellular cleavage of Notch receptors that is necessary for the activation of Notch signaling (7). The 6 PSENEN mutations comprised: (a) 2 splice site mutations, c. $62-1 \mathrm{G}>\mathrm{C}$ and g.1412T $>\mathrm{C}$ ); (b) 2 nonsense mutations, c.35T $>\mathrm{A}(\mathrm{p}$. Leu12*) and c.115C $>\mathrm{T}$ (p.Arg39*); and (c) 2 frameshift mutations, c.84_85insT (p.Leu29Serfs ${ }^{*}$ 2) and c.216delC (p.Ser73Profs ${ }^{*} 72$ ) (Figure $1 \mathrm{~K}$ and Supplemental Figure 3). All sequence deviations resulted in null alleles and cosegregated with the disease phenotype in the affected families. They were absent in dbSNP137, the 1000 Genomes project database, Exome Variant Server, and ExAC.

In silico analysis of the splice site mutation c. $62-1 \mathrm{G}>\mathrm{C}$ revealed disruption of the WT acceptor site in exon 2 (Supplemental Figure 4). This resulted in either the use of an alternative splice site further downstream or exon skipping. Since no RNA samples from patients were available, exon trapping was used to evaluate the structural consequences of this mutation. The results revealed complete skipping of exon 2, leading to an in-frame deletion of 35 aa within the cytoplasmic domain of PEN-2 (Supplemental Figure 5, A and B). For the splice site mutation g.1412T $>C$, RNA from the mutation carrier was available. Sequencing of transcribed cDNA showed skipping of exon 2 (Supplemental Figure 5, C and D).

The nonsense and frameshift mutations led to alternative stop codons. To examine the consequences of these changes, WT PEN-2 and the mutants p.Arg $39^{*}$ and p.Ser73Profs ${ }^{*} 72$ were fused to a FLAG epitope and overexpressed in HEK293T cells. These experiments showed that the WT construct led to translation of a protein of approximately $12 \mathrm{kDa}$ in size, consistent with previous reports on PEN-2 (8). We detected no protein for either mutant (Figure 2C). We then blocked the proteasome using MG-132, thereby demonstrating that mutation p.Ser73Profs ${ }^{*} 72$ led to an elongated mutant protein of approximately $15 \mathrm{kDa}$ in size (Figure 2D), suggesting the translation of an unstable mutant protein that would be subject to rapid degradation by the proteasome complex. In contrast, no protein translation was observed for p.Arg39*, even after proteasome blocking. These data strongly suggest that the detected PSENEN mutations cause haploinsufficiency of PEN-2, through either proteasomal degradation or mRNA decay.

To provide in vivo evidence for the role of PSENEN mutations in DDD, we performed knockdown of the PSENEN homolog psenen in zebrafish larvae using antisense morpholinos (Supplemental Figure 1). At both 72 and 96 hours post fertilization (hpf), we observed a major decrease in pigment cells in zebrafish larvae injected with the pen-2 antisense morpholino (MO1 and MO2 groups) compared 


\section{Table 1. Clinical features of patients with PSENEN mutations}

\begin{tabular}{|c|c|c|c|c|c|c|c|c|c|c|}
\hline \multirow{3}{*}{$\begin{array}{l}\text { Mutation } \\
\text { Ethnicity } \\
\text { Sex }\end{array}$} & \multicolumn{2}{|c|}{ c. $35 T>A$} & \multirow{3}{*}{$\begin{array}{c}\text { c.115C>T } \\
\text { German }\end{array}$} & \multicolumn{3}{|c|}{ c. $62-1 \mathrm{G}>\mathrm{C}$} & \multicolumn{2}{|c|}{ 84_85insT } & \multirow{2}{*}{$\begin{array}{c}\text { c.216delC } \\
\text { German }\end{array}$} & \multirow{2}{*}{$\begin{array}{c}\text { g.1412T>C } \\
\text { French }\end{array}$} \\
\hline & \multicolumn{2}{|c|}{ German } & & \multicolumn{3}{|c|}{ Indian } & \multicolumn{2}{|c|}{ Thai } & & \\
\hline & M & $\mathrm{F}$ & & $\mathrm{F}$ & M & $\mathrm{F}$ & $M$ & $\mathrm{~F}$ & $\mathrm{~F}$ & $\mathrm{~F}$ \\
\hline \multicolumn{11}{|l|}{ DDD } \\
\hline Face & NA & NA & NA & + & + & + & + & + & + & - \\
\hline Trunk & NA & NA & NA & + & + & + & + & + & + & - \\
\hline Axillae & NA & NA & NA & + & + & + & + & + & + & + \\
\hline Inframammary region & NA & NA & NA & NA & + & + & NA & + & + & + \\
\hline Neck & NA & NA & NA & + & + & + & + & + & + & + \\
\hline Inguinal folds & NA & NA & NA & NA & + & NA & + & + & + & + \\
\hline DDD histology & + & NA & + & + & + & NA & + & NA & + & + \\
\hline FHK & + & NA & + & + & + & NA & + & NA & + & + \\
\hline \multicolumn{11}{|l|}{ Al } \\
\hline Al & + & + & + & - & + & + & - & - & - & + \\
\hline Obesity & + & + & + & - & + & + & - & - & - & + \\
\hline Nicotine abuse & + & + & + & - & - & - & - & - & - & - \\
\hline Axillae & NA & NA & NA & - & + & + & - & - & - & + \\
\hline Inframammary region & NA & NA & NA & - & - & - & - & - & - & + \\
\hline Inguinal folds & + & NA & NA & - & + & - & - & - & - & + \\
\hline Cenitals & + & NA & NA & - & + & - & - & - & - & - \\
\hline
\end{tabular}

+ or -, denotes the presence or absence of a clinical feature, respectively; NA, clinical information was not available; F, female; M, male.

with pigment cell numbers in the 2 control groups. The control groups comprised: (a) zebrafish larvae injected with pen-2 scrambled morpholino (CoMO1 and CoMO2 groups); and (b) untreated zebrafish larvae (WT group) (Figure 3A and Supplemental Figure 6). These findings are consistent with those of a previous study, which reported a reduction in melanin content for pofut1 morpholino knockdown in zebrafish (5). Interestingly, besides a general decrease in pigmentation, the present knockdown zebrafish larvae showed substantial alterations in the distribution of pigmentation compared with that seen in the control groups, particularly in the facial and tail regions. This apparently haphazard distribution of pigment cells rendered the MO group easily distinguishable from the control groups. In addition, whereas the MO group displayed significantly fewer pigment cells in the dorsal area of the head, hyperpigmentation of the tail fins was present (Figure 3, B and C).
These pigmentation abnormalities can be considered the phenotypic counterpart of human DDD and were absent in the control groups. Next, we performed in vivo life imaging of developing zebrafish larvae, with a focus on the migration and differentiation of pigment cells. In the CoMO group, we observed an organized, target-oriented migration of pigment cells, resulting in the typical larvae stripe pattern. In contrast, we observed a disordered and meandering migration of pigment cells in the MO group, resulting in a haphazard distribution of pigment (Figure 3D). The differentiation of pigment cells was monitored by evaluating their shaping. All pigment cells in the CoMO group were regular in size and showed a similar flat and elongated morphology. In contrast, while consistent in terms of their morphology, the pigment cells in the MO group were irregular in size (Figure 3, E and F). This observation is consistent with previous electron microscopy investigations of
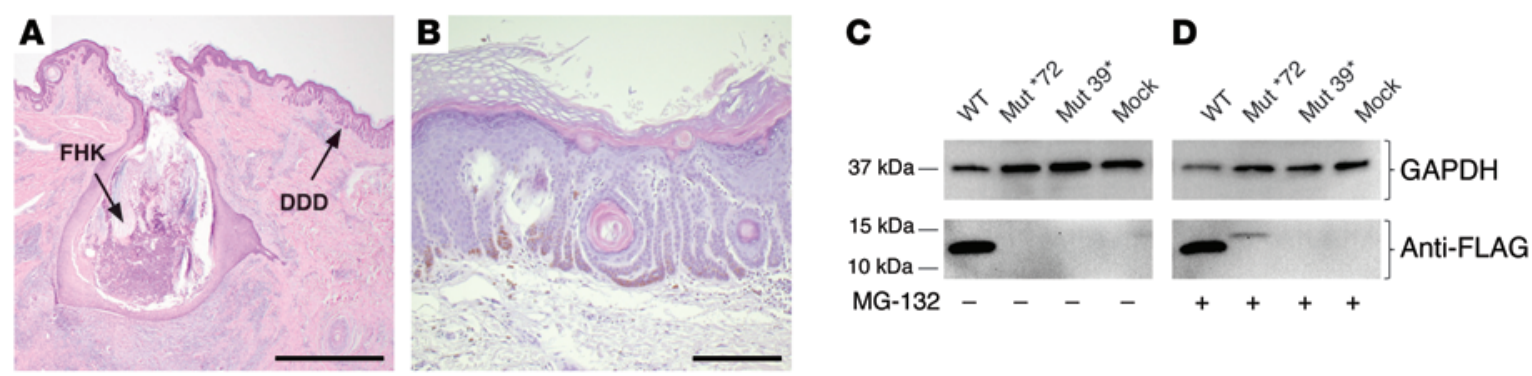

Figure 2. Dermatohistological examination of PSENEN mutation carriers and immunoblotting results. (A and B) Histological examination revealed digitiform reteacanthosis, pronounced hyperpigmentation at the tips of the rete ridges, small horn cysts, and pronounced follicular hyperkeratosis (FHK). Scale bars: 4,000 $\mu \mathrm{m}$ (A); $200 \mu \mathrm{m}$ (B). (C and D) Immunoblotting of cell lysates following transient transfection with WT PSENEN and PSENEN-mutant (Mut * 72, Mut 39*) constructs. (C) Bands of the expected 12-kDa size were detected for WT PEN-2. No bands were detected for the 2 mutated constructs. $n=3$. (D) Blocking of the proteasome with MG-132 led to detection of an elongated PEN-2 protein (approximately 15 kDa in size), resulting from the mutation c.216delC (p.Ser73Profs*72). No protein was detected for the c.115C>T-mutation (p.Arg39*). $n=3$. 

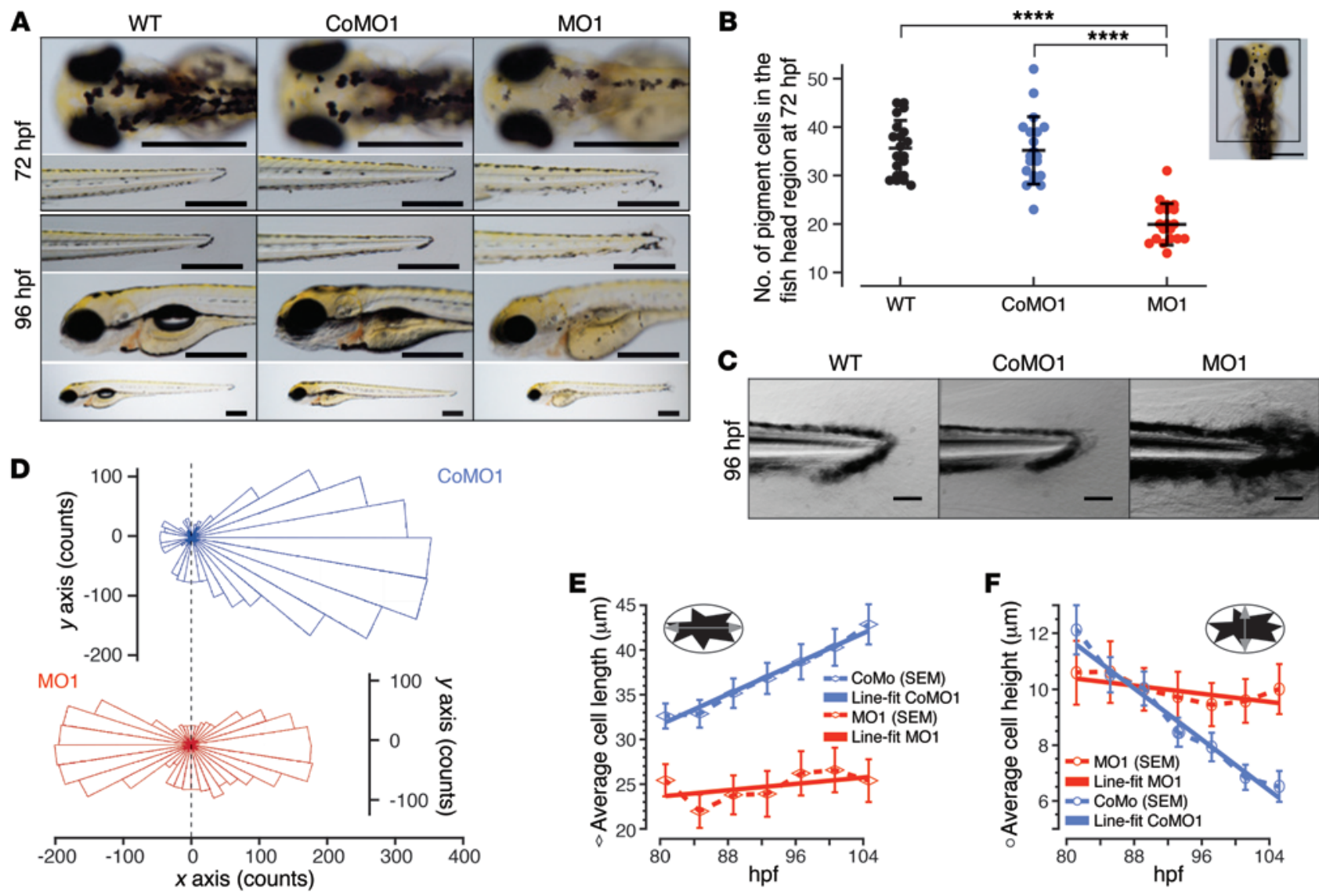

Figure 3. Knockdown of PSENEN homolog psenen in zebrafish. Results are shown for noninjected WT and CoM01- and M01-injected zebrafish larvae. (A) Representative phenotype observations at 72 and $96 \mathrm{hpf}$. In the M01 group, a disordered and abnormally located distribution of pigment was observed. Additional pigment cells were observed in the lateral facial and tail fin regions. Both features were absent in the control groups. Images are representative of 5 independent experiments, with more than 100 larvae analyzed per group. Scale bars: $500 \mu \mathrm{m}$. (B) Dot plot showing the average distribution of pigment cell numbers in the zebrafish larvae dorsal head region (see inset) of the M01 group compared with the distribution in both control groups. $n=20$ samples per group from 3 independent experiments. Scale bar: $500 \mu \mathrm{m}$. ${ }^{* * *} P \leq 0.0001$, by 1-way ANOVA with Bonferroni's post-hoc test for multiple comparisons. (C) Representative tail fin pigmentation phenotype, showing the maximum projection of recorded and overlaid random tail fin images from the MO group and both control groups, respectively. $n=10$ images per group from 3 independent experiments. Scale bars: $125 \mu$ m. (D) Rose plot showing the directional distribution of single consecutive migration steps of multiple individual pigment cells in M01- and CoM01-injected zebrafish larvae. M01: $n=74$ cells from 3 independent experiments; CoM01: $n=72$ cells from 3 independent experiments. (E and $\mathbf{F}$ ) Curve charts showing the shaping of pigment cells in developing zebrafish larvae (see inset illustrations). M01: $n=44$ cells from 3 independent experiments; CoM01: $n=54$ cells from 3 independent experiments. Data represent the mean \pm SEM. CoMo, control morpholino.

DDD-affected skin, which revealed irregular cell shapes and sizes (4). Given these findings, we hypothesized that the underlying pathomechanisms of DDD involve disordered migration of melanocytic precursor cells into the epidermis and irregular differentiation of epidermal melanocytes.

PEN-2 forms, together with presenilin, nicastrin, and anterior pharynx defective, the $\gamma$-secretase protein complex. $\gamma$-Secretase plays a key role in the Notch pathway, where it catalyzes the intramembranous cleavage of type I membrane proteins, including Notch and amyloid precursor protein (7). The Notch pathway is crucial for developmental processes, and, in the skin, Notch signaling is thought to mediate interactions between melanocytes and keratinocytes, thereby regulating the delicate balance between the proliferation and differentiation of these cells (9).

Involvement of this pathway in pigmentation processes was primarily suggested by murine-KO models. For instance, mice lacking recombination signal-binding protein for immunoglob- ulin $\kappa \mathrm{J}$ region (RBPJ), which encodes a transcriptional factor of the Notch pathway, as well as NOTCH1- and NOTCH2-deficient mice exhibit premature graying of the hair $(9,10)$. Interestingly, in these mouse models, hypopigmentation was described in the follicular epidermis and hair coat, while it is not clear whether the interfollicular epidermis was examined. By contrast, in humans, mutations in distinct Notch pathway genes lead to a cutaneous hyperpigmentation phenotype, with hyperpigmentation of the interfollicular epidermis as the result of a Notch signaling disturbance in patients with DDD $(5,6)$. These differences in pigmentation suggest that aberrant Notch signaling may have cell typespecific effects on different components of the skin.

Aberrant pigmentation due to alterations in Notch signaling might be explained by abnormal migration of melanoblasts and melanocytes, as supported by (a) our observations in the psenen-knockdown zebrafish and (b) the presence of melanoblasts and melanocytes in unusual ectopic locations of the dermal papil- 
la and the skin in $R b p j-K O$ mice (10). Still, mechanisms other than migration could be involved in the pathogenesis of DDD, as suggested above. For example, Rbpj-KO mice also demonstrate the importance of Notch signaling in regulating the differentiation of melanoblasts into melanocytes, suggesting that alterations in the proliferation and differentiation dynamics of melanoblasts can also confer abnormal pigmentation (10). Further, little is known about the exact mechanisms and the pathways involved in the distribution and transport of melanosomes from melanocytes into keratinocytes. Alterations in these processes caused by deficiencies in Notch pathway members such as PEN-2, POGLUT1, and POFUT1 may also contribute to the pathogenesis of DDD, although no studies have thus far addressed this aspect. In this context, it is intriguing that KRT5 has been shown to be involved in melanosome transfer (4), thus indicating that there might be a crosstalk between Notch and KRT5 signaling. Therefore, further elucidation of the exact pathogenetic mechanisms through which Notch signaling orchestrates pigmentation processes is of crucial interest.

Previously, 3 mutations in PSENEN were reported in a small subset of familial AI (11), a chronic, relapsing inflammatory hair follicle disorder. Pathogenetically, follicular hyperkeratosis with closure of the upper part of the hair follicle and an accompanying lymphocytic infiltration were implicated (12). Interestingly, 6 individuals with DDD from the present cohort had AI, and all reported nicotine abuse and/or obesity. Even more interestingly, histopathological examination in these individuals also revealed follicular hyperkeratosis in body areas with reticulate hyperpigmentation (Figure 2, A and B), suggesting that this histological feature could serve as a prospective marker to identify DDD patients with susceptibility to AI.

Although no DDD symptoms were reported for the 3 previous AI patients with PSENEN mutations, axillary and inguinal hyperpigmentation has been described in 2 of them (11). This raises the question of whether a comorbid diagnosis of DDD was overlooked, particularly since a phenotypic overlap between DDD and AI has been reported. Notably, this clinical overlap is particularly apparent in the predilection sites, namely the back, buttocks, and intertriginous areas (13). Thus, the present report is the first to our knowledge to demonstrate a molecular genetic link between DDD and AI comanifestation.

We hypothesize that the majority of deleterious PSENEN mutation carriers present primarily with DDD, while those with a history of nicotine abuse or obesity have an increased susceptibility to comorbid AI. This hypothesis is supported by Loo et al., who stated that the follicular anomaly inherent in DDD may predispose individuals to develop AI (14).

Here, we show that PSENEN mutations underlie a previously undescribed subtype of DDD, which is clinically and histopathologically distinct from the previously reported subtypes. Impor- tantly, this subtype is associated with an increased susceptibility to $\mathrm{AI}$ in the presence of particular risk factors. This hypothesis is supported by our mammalian cell culture experiments and studies based on psenen knockdown in zebrafish. From a translational perspective, the co-occurrence of DDD and AI renders the detection of PSENEN mutations that result in null alleles highly relevant, since these findings could facilitate the development of therapeutic strategies for AI. The present data contribute to an improved understanding of the complex biology of diseases caused by a disturbance of Notch metabolism and emphasize the crucial role of this pathway in pigmentation and differentiation of the epidermis.

\section{Methods}

Further information is provided in the Supplemental Methods and Supplemental Tables 1-7.

Statistics. After confirming Gaussian distribution by the D'Agostino-Pearson omnibus test, the statistical analyses shown in Figure 3C were performed using 1-way ANOVA followed by Bonferroni's posthoc test for multiple comparisons. A $P$ value of less than 0.05 was considered statistically significant.

Study approval. Approval for this study was obtained from the ethics committees of the medical faculties of the University of Düsseldorf and the University of Bonn. All participants provided written informed consent prior to blood sampling. All participants written informed consent was provided for use of the photographs in this article. This study was conducted in accordance with Declaration of Helsinki principles.

\section{Author contributions}

RCB initiated and oversaw the study. DJR, FBUB, JF, BO, and RCB coordinated the work and prepared the manuscript. J. Wititsuwannakul, S. Delker, S. Danda, UH, SAP, DL, and J. Wenzel clinically examined the patients and collected blood samples. HT, JA, and PN performed whole-exome sequencing. DJR, AT, SW, MB, and BO performed the experiments.

\section{Acknowledgments}

We thank the patients and families for participating in this study. This work was supported by local funding (Forschungsförderprogramm der Medizinischen Fakultät der Universität Bonn [BONFOR], to DJR and RCB). BO is funded by a repatriation grant from the North Rhine-Westphalian Ministry of Innovation, Science, and Research. RCB is a member of the Deutsche Forschungsgemeinschaft-funded (DFG-funded) Excellence Cluster ImmunoSensation and was a recipient of a Heisenberg Professorship from the DFG (BE 2346/4-2).

Address correspondence to: Regina C. Betz, Institute of Human Genetics, Sigmund-Freud-Str. 25, D-53127 Bonn, Germany. Phone: 49.0.228.28751023; E-mail: regina.betz@uni-bonn.de.
1. Baxter LL, Pavan WJ. The etiology and molecular genetics of human pigmentation disorders. Wiley Interdiscip Rev Dev Biol. 2013;2(3):379-392.

2. Dowling GB, Freudenthal W. Acanthosis nigricans. Proc R Soc Med. 1938;31(9):1147-1150.

3. Degos R, Ossipowski B. Dermatose pigmentaire réticulée des plis. Ann Dermatol Syphiligr.
1954;81(2):147-151.

4. Betz RC, et al. Loss-of-function mutations in the keratin 5 gene lead to Dowling-Degos disease. Am J Hum Genet. 2006;78(3):510-519.

5. Li M, et al. Mutations in POFUT1, encoding protein $\mathrm{O}$-fucosyltransferase 1 , cause generalized Dowling-Degos disease. Am J Hum Genet.
2013;92(6):895-903

6. Basmanav FB, et al. Mutations in POGLUT1, encoding protein $\mathrm{O}$-glucosyltransferase 1 , cause autosomal-dominant Dowling-Degos disease. Am J Hum Genet. 2014;94(1):135-143.

7. Bergmans BA, De Strooper B. $\gamma$-secretases: from cell biology to therapeutic strategies. Lancet Neu- 
rol. 2010;9(2):215-226.

8. Prokop S, Haass C, Steiner H. Length and overall sequence of the PEN-2 C-terminal domain determines its function in the stabilization of presenilin fragments. J Neurochem. 2005;94(1):57-62.

9. Kumano K, et al. Both Notch1 and Notch2 contribute to the regulation of melanocyte homeostasis. Pigment Cell Melanoma Res. 2008;21(1):70-78.
10. Aubin-Houzelstein G, et al. Melanoblasts' proper location and timed differentiation depend on Notch/RBP-J signaling in postnatal hair follicles. J Invest Dermatol. 2008;128(11):2686-2695.

11. Pink AE, et al. PSENEN and NCSTN mutations in familial hidradenitis suppurativa (Acne Inversa). J Invest Dermatol. 2011;131(7):1568-1570.

12. Jemec GB. Clinical practice. Hidradenitis suppu- rativa. $N$ Engl J Med. 2012;366(2):158-164.

13. Bedlow AJ, Mortimer PS. Dowling-Degos disease associated with hidradenitis suppurativa. Clin Exp Dermatol. 1996;21(4):305-306.

14. Loo WJ, Rytina E, Todd PM. Hidradenitis suppurativa, Dowling-Degos and multiple epidermal cysts: a new follicular occlusion triad. Clin Exp Dermatol. 2004;29(6):622-624. 